\title{
Valeur de l'immunité conférée par un vaccin antipéripneumonique lyophilisé préparé à l'aide de la souche $T_{1}$
}

\author{
par M. P. DOUTRE et J. CHAMBRON
}

\begin{abstract}
RESUME
Au cours des années 1968 et 1969 , une série d'épreuves tendant à apprécier la valeur de l'immunité conférée par un vaccin lyophilisé préparé à laide de la souche $T_{1}$ (44 passage) a été effectuée au Laboratoire de Dakar. La méthode dite de contact, mise au point par les chercheurs australiens, a été retenue. Trois mois et sept mo1s et demi après la vacciration, l'immunité est totale; quatorze mois après l'immunisation, elle est encore de 90 p. 100. Dans ces conditions, une période de protection munmale de un an peut être garantie.
\end{abstract}

A la suite des recommandations de la Conférence des Experts de la péripneumonie bovine tenue à Khartoum en février 1967, et des possibilités de mise au point de campagnes inter-états de vaccination contre cette maladie, le service de bactériologie du Laboratoire national de l'Elevage et de Recherches vétérinaires du Sénégal a mené au cours des années 1967, 1968 et 1969 une série d'expériences destinées à apprécier la valeur de l'ımmunité conférée par différents vaccins lyophilisés. Un premier travail concernant la souche $\mathbf{K H}_{3} \mathrm{~J}$ (86 ${ }^{\mathrm{C}}$ passage) a déjà fait l'objet d'une précédente publication (8). Le présent article se propose de rapporter les résultats obtenus avec un vaccin préparé à laide de la souche $T_{1}$ (44 passage). Les épreuves ont eu lieu respectivement trois mois, sept mois et demi et quatorze mois après la vaccination. Ces temps correspondent à la période s'écoulant entre la date de la vaccination et la mise en contact d'un lot de vaccinés avec des bovins infectants et témoins. Les animaux utilisés appartenaient à la race Ndama, race bovine africaine particulièrement sensible à la péripneumonie. Ils provenaient de régions indemnes de péri- pneumonie depuis de nombreuses années et n'avaient jamais été vaccinés.

\section{VACCIN UTILISE}

Il résulte de la lyophilisation d'un mélange à volume égal d'une culture de Mycoplasma mycoides souche $\mathbf{T}_{1}$ (44* passage) et de mist dessicans $\left({ }^{*}\right)$.

La composition du milieu de culture résulte des travaux effectués au Laboratoire de FortLamy (milieu F 66, rapport présenté par A. PROVOST à la $3^{\text {e }}$ Réunion des Experts sur la péripneumonie bovine F.A.O./O.I.E./O.A.U. tenue à Khartoum en février 1967 (13) et de ceux des chercheurs australiens du C.S.I.R.O.

Quelques modifications ont été apportées; elles portent sur la suppression de l'acide palmitique pratiquement insoluble même dans une

("i) Actuellement, à Dakar, seul le lait écrémé sec est utilisé comme support de lyophilisation dans la préparation des vaccins lyophilisés contre la péripneumonie bovine. 
solution de soude $0,1 \mathrm{M}$, et sur la préparation et la quantité d'extralt frais de levure ajouté :

- macération de cocur: on provoque la macération pendant une heure dans un litre d'eau à $50^{\circ} \mathrm{C}$ de $500 \mathrm{~g}$ de hachis de cour de bœuf dégraissé, puis on porte à ébullition. Filtration à chaud sur papier. On ajoute par litre: $10 \mathrm{~g}$ de tryptose (Difco), $2 \mathrm{~g}$ de glucose et $2,5 \mathrm{~g}$ de phosphate disodique. On chauffe à $80^{\circ} \mathrm{C}$; filtration à chaud.

- addition de :

Glycérol

Acide oléique

Extrait frais de levure

$15 \mathrm{~g} / 1$

$15 \mathrm{mg}$

Sérum de cheval décomplémenté $100 \mathrm{ml}$

Pénicilline

1.000 .000 unités

Le pH est amené à 8,1 avec une solution de soude $10 \mathrm{M}$. L'acide oléique est mélangé à $5 \mathrm{ml}$ de soude $0,1 \mathrm{M}$; le gel formé est dissout par agitation magnétique.

- extrait de levure : l'extrait de levure préparé extemporanément présente des qualités supérieures aux produits correspondants que l'on trouve dans le commerce. La technique de préparation retenue est la suivante (I.E.M.V.T.laboratoire de microbiologie) :

- mettre en suspension homogène $1 \mathrm{~kg}$ de levure fraîche de boulangerie dans un litre d'eau distillée en présence de $8 \mathrm{ml}$ de chloroforme;

- laisser au bain-marie 24 heures à $50^{\circ} \mathrm{C}$ en agitant de temps en temps; centrifuger et recueillir un premier surnageant que l'on conserve au froid;

- reprendre les culots dans un litre d'eau distillée et les soumettre à quatre cycles geldégel;

- centrifuger à nouveau et recueillir le deuxième surnageant;

- mélanger les deux surnageants;

- filtrer sur filtre Seitz EKS 1, conservation à $-20^{\circ} \mathrm{C}$.

Pour la préparation du vaccin, on utilise une culture de 72 heures à $37^{\circ} \mathrm{C}$. Au cours des dernières 24 heures, les ballons sont portés sur agitateur magnétique.

La répartition se fait sous un volume de $5 \mathrm{ml}$ par flacon type pénicilline de $20 \mathrm{ml}$.
Au moment de l'emploi, le contenu de chaque flacon est reconstitué avec $20 \mathrm{ml}$ d'eau distillée stérile froide (20 doses vaccinales). Dans ces conditions, le titrage des vaccins par la méthode des dilutions donne un minimum de $10^{9}$ unités viables par $\mathrm{ml}$. Ce titre est égal à celui des meilleurs lots de vaccin-culture mesuré au moment de leur conditionnement.

\section{VACCINATION DES ANIMAUX}

Le 10 juillet 1968, 50 bovins Ndama ont été vaccinés $(1 \mathrm{ml}$ de vaccin reconstitué, inoculé à la côte) et conservés à la ferme de Sangalkam. Ils ont donné lieu à la constitution de trois lots, utilisés respectivement 3 mois, 7 mois et demi et 14 mois après la vaccination.

A la suite de la vaccination, environ 50 p. 100 des animaux ont présenté une réaction locale au point d'inoculation qui ne dépassa jamais la taille de la main. La presque totalité des sujets immunisés a offert une réaction sérologique (déviation du complément Kolmer) d'intensité variable selon les sujets. A titre indicatif, le tableau 1 indique l'évolution sérologique post-vaccinale des animaux vaccinés ayant été utilisés lors de la première phase de l'expérience (immunité 3 mois). On peut voir que le $\mathrm{n}^{\circ} 25$ qui n'a présenté aucune réaction locale fixe le complément au 1/160, 28 jours après la vaccination.

\section{EPREUVE D'IMMUNITE}

\section{a) Principe de la méthode}

Les animaux vaccinés ont été éprouvés en utilisant la méthode de mise en contact étroit décrite par les auteurs australiens. Cette technique consiste à mettre en présence, dans une enceinte close de volume restreint, un lot d'animaux vaccinés, un lot d'animaux témoins non vaccinés et un lot de bovins infectés expérimentalement par voie endobronchique. Pour que l'expérience soit concluante, dans l'absolu, il convient qu'en fin d'expérience les bovins intubés et les témoins non vaccinés succombent de péripneumonie alors que les animaux vaccinés résistent et présentent à l'autopsie un appareil pulmonaire indemne de toute atteinte péripneumonique. 
Tableau $n^{\circ} 1$ - Sérologie post-vaccinale des animaux vaccinés avec $T_{1}$ avant la mise en contact (dêriation du complëment Kolmer)

\begin{tabular}{|c|c|c|c|c|c|c|}
\hline $\begin{array}{l}N^{\circ} \text { des } \\
\text { antmatux }\end{array}$ & $\begin{array}{c}\text { Vaccination } \\
s_{0}\end{array}$ & $J_{0}+13$ & $\mathrm{~J}_{0}+20$ & $\mathrm{~J}_{0}+28$ & $\mathrm{~J}_{0}+41$ & $\begin{array}{l}\text { Réaction locale au } \\
\text { point d'incculation }\end{array}$ \\
\hline 25 & - & $1 / 5$ & $1 / 40$ & $1 / 160$ & $1 / 80$ & - \\
\hline 24 & - & $1 / 5$ & $1 / 40$ & $1 / 40$ & $1 / 20$ & - \\
\hline 9 & - & - & - & $\rightarrow$ & - & - \\
\hline 42 & - & $1 / 20$ & $1 / 10$ & $1 / 20$ & $1 / 10$ & - \\
\hline 83 & - & - & - & - & - & - \\
\hline 10 & - & $1 / 10$ & $1 / 10$ & - & - & - \\
\hline 79 & - & $1 / 5$ & $1 / 20$ & $1 / 10$ & $1 / 5$ & - \\
\hline $1 I \mathrm{~B}$ & - & $1 / 5$ & $1 / 10$ & $1 / 10$ & $1 / 5$ & $\begin{array}{l}+++(\text { taille de la } \\
\text { main) }\end{array}$ \\
\hline 80 & - & $1 / 10$ & $1 / 80$ & $1 / 160$ & $1 / 320$ & +++ \\
\hline 90 & - & $1 / 10$ & $1 / 40$ & $1 / J 60$ & $1 / 160$ & +++ \\
\hline 11 & - & $1 / 10$ & $1 / 40$ & $1 / 80$ & $1 / 20$ & +++ \\
\hline 82 & - & $1 / 10$ & $1 / 40$ & $1 / 10$ & $1 / 10$ & ++ \\
\hline 2 & - & $1 / 5$ & $1 / 5$ & $1 / 5$ & - & ++ \\
\hline $18 B$ & - & - & $1 / 10$ & $1 / 20$ & $1 / 10$ & +++ \\
\hline 93 & - & $1 / 5$ & $1 / 10$ & $1 / 5$ & - & +++ \\
\hline
\end{tabular}

\section{b) Bâtiment}

En tenant compte des dimensions relatives du bâtiment construit par les chercheurs australiens du C.S.I.R.O., une étable a été spécialement aménagée dans la ferme annexe du Laboratoire à Sangalkam pour réaliser les épreuves d'immunité par la méthode de contact. Actuellement, ses dimensions sont les suivantes:

- longueur: $10 \mathrm{~m}$

- largeur : $10 \mathrm{~m}$

- hauteur: $3 \mathrm{~m}$

Pour assurer l'alimentation des animaux, quatre mangeoires de $5 \mathrm{~m}$ de long sont placées de chaque côté. Le foin stocké sur le fauxplafond en planches est directement descendu dans les mangeoires grâce à des trappes aménagées à cet effet.

L'aération est assurée par onze ouvertures de $1 \mathrm{~m} \times 0,50 \mathrm{~m}$, situées à $2,35 \mathrm{~m}$ du sol.

A l'extérieur, un parc de $120 \mathrm{~m}^{2}$ pourvu d'un abreuvoir permet de sortir les animaux chaque matin pendant environ une demi-heure.
Une fois par semaine, lors des prises de sang, ce temps est prolongé de deux ou trois heures.

\section{c) Alimentation des animaux}

$\mathrm{Au}$ cours des différentes épreuves, les bovins ont été alimentés suivant la saison et les disponibilités avec:

- soit du foin et de la paille naturels;

- soit du maïs fourrager;

-- soit du sorgho ensilé ( $S$. vulgare);

- soit un concentré en granulés constitué par des sons de froment, de sorgho et de maïs;

- ou un aliment composé, préparé au Laboratoire, comprenant des coques darachide mélassées à 20 p. 100 auxquelles sont adjoints de la farine de riz et du son de mais $(*)$.

() CALVET (H), VALENZA (J.) et BOUDERGUES (R.). Coque d'arachide et alimentation du. bétai] Colloque OCAM sur l'Elevage, Fort-Lamy, 8-13 décembre 1969, CE-FL $\Omega^{\circ} 15$, sect. 4-3.

VALENZA (J.), CALVET (H.), BOUDERGUES (R.) et ORUE (J.). Essais d'embouche intensive de zćbus peulh sénégalais (Gobra). Colloque OCAM sur 1'Elevage, Fort-Lamy, 8-13 décembre 1969, CE-FL $\pi^{\circ} 19$, sect. $4-1$. 


\section{A. VALEUR DE L'IMMUNITE TROIS MOIS APRES LA VACCINATION}

\section{Intubation des animaux destinés à devenir infectants}

\section{Inoculum}

Après anesthésie en chloral intraveineux, chaque animal reçoit une injection intratrachéale administrée à la sonde, suivant la technique devenue classique, de $20 \mathrm{ml}$ d'un mélange à parties égales de :

- broyat de lésions pulmonaires péripneumoniques,

- lymphe péripneumonique,

_- bouillon cour sérum (milieu F 66 modifié).

Les lésions pulmonaires et la lymphe péripneumonique sont récoltées auparavant sur un malade sacrifié sur le terrain au dernier stade de la maladie, et conservées jusqu'au moment de l'emploi à $-20^{\circ} \mathrm{C}$.

Un isolement de $M$. mycoides à partir des lésions pulmonaires et de la lymphe est effectué au moment de la préparation de l'inoculum pour confirmer la qualité du matériel utilisé.

Pour la première phase de l'expérimentation, deux lots d'animaux ont été successivement rendus infectants. En effet, la mortalité très rapide des animaux du premier lot a fait craindre la possibilité d'une mauvaise contamination des témoins et des vaccinés. Un second lot de bovins infectants a donc été préparé. Ces animaux ont été introduits 21 jours après le début de la mise en contact; il ne restait alors plus que trois bovins infectants survivants du premier lot.
$\mathrm{N}^{\text {wos }}$ des bovins infectants du $1^{\mathrm{er}}$ lot (15 bovins)

$\mathrm{N}^{\mathrm{os}}$ des bovins infectants du $1^{\text {er }}$ lot (12 bovins)
$13,88,32,44,36,29$,

$14,26,56,23,57,15$, $18,76,95$

$702,709,710,721,722$, $724,715,34,717,716$, 711,718

\section{Mise en contact étroit des animaux vaccinés, des témoins non vaccinés et des intubés}

Trois mois après la vaccination, quinze bovins vaccinés ont été groupés avec quinze bovins infectants ( $1^{\mathrm{cr}}$ lot) en présence de quinze témoins ( $\mathrm{n}^{\text {ms }} 75,43,38,40,22,31,50,3,63 \mathrm{~B}$, $41,5 \mathrm{~B}, 4,91,20,74)$. Le deuxième lot de bovins infectants a ćté introduit dans l'étable expérimentale 21 jours après le début de la mise en contact.

\section{Evolution des animaux}

Les observations s'arrêtent 94 jours après le début de la mise en contact.

\section{a) Evolution des animaux infectants}

Seule l'évolution sérologique est rapportée dans les tableaux qui suivent. Le devenir des anticorps fixant le complément a été suivi par la méthode de KOLMER. La recherche de l'antıgène circulant a été effectuée par précipito-diffusion en boîte de Pétri (gélose noble à 1 p. 100 dissoute dans un tampon véronal à pH $7,3-7,4$, merthiolaté à 0,04 p. 100). Le sérum anti- $M$. mycoides a été préparé par hyperimmunisation de moutons.

Les résultats obtenus figurent dans les tableaux $2,3,4$ et 5 ( $1^{\text {er }}$ et $2^{\text {e }}$ lots d'animaux infectants). En matière de déviation du complément, seules les réactions ++++ ont ćté retenues.

Sur 27 bovins infectés par voie endobronchique, 23 ont succombé en présentant à l'autopsie des lésions péripneumoniques et 4 ont été sacrifiés en fin d'expérience. Le maximum des mortalités dues à la maladie expérimentale se situe vers le $20^{\circ}$ jour qui suit l'intubation. Chez les survivants, la présence de séquestres encapsulés a été constatée chez deux sujets (76 et 724). Le poumon était totalement indemne chez les deux autres (13 et 36 ). Le 36 fixait le complément à un taux élevé; de plus l'antigène circulant a été décelé entre le $35^{\mathrm{e}}$ et le $75^{\mathrm{e}}$ jour. Ces deux animaux, sérologiquement négatifs au départ, présentaient une immunité certaine. Dans ce cas, l'évolution sérologique ne semble traduire que la réaction de l'organisme à l'introduction d'une substance antigénique étrangère en quantité importante.

\section{b) Evolution des témoins}

L'évolution sérologique fait l'objet des tableaux 6 et 7 (déviation du complément, recherche de l'antigène circulant).

Sur 15 témoins, 11 sont morts de péripneumonie bovine entre le $35^{\mathrm{e}}$ et le $63^{\circ}$ jour 
Tableau 2 - Evolution sérologique des avimaxx intubés du premer lot (Dévlation du complément Kolmer)

\begin{tabular}{|c|c|c|c|c|c|c|c|c|c|c|c|c|c|c|}
\hline $\begin{array}{c}\text { Nombre de jours apres } \\
1 \text {, intubation }\end{array}$ & 7 & 23 & 21 & 28 & 55 & 41 & 49 & 56 & 63 & 70 & 77 & 84 & 91 & $\begin{array}{c}\text { Abattage } \\
\text { log }\end{array}$ \\
\hline $\begin{array}{c}\mathrm{N}^{0} \text { des animaux } \\
13\end{array}$ & $1 / 10$ & $1 / 320$ & $=/ 320$ & $1 / 160$ & $1 / 80$ & $1 / 40$ & $1 / 40$ & $1 / 40$ & $1 / 10$ & $1 / 10$ & $1 / 5$ & $1 / 5$ & $1 / 5$ & $\begin{array}{l}\text { Absence lésion pér1- } \\
\text { |pneunnor.1que }\end{array}$ \\
\hline 88 & $1 / 20$ & $1 / 160$ & $1 / 320$ & $\begin{array}{c}\bar{E} \cdot \bar{E} \cdot \mathrm{B} \\
+ \\
\end{array}$ & & & & & & & & & & \\
\hline 32 & $1 / 40$ & $\begin{array}{c}\text { I.P.P } \\
+\end{array}$ & & & & & & & & & & & & \\
\hline 44 & - & $1 / 320$ & $\begin{array}{l}\text { L.P.B } \\
+\end{array}$ & & & & & & & & & & & \\
\hline 36 & $1 / 5$ & $1 / 80$ & $1 / 320$ & $1 / 640$ & $1 / 320$ & $1 / 80$ & $1 / 80$ & $1 / 80$ & $1 / 40$ & $1 / 20$ & $1 / 20$ & $1 / 20$ & $1 / 20$ & $\begin{array}{l}\text { Absence lésion DérI- } \\
\text { pneunon } q \text { que }\end{array}$ \\
\hline 29 & - & $1 / 80$ & $\begin{array}{c}\mathrm{L} . \mathrm{P.3} \\
+\end{array}$ & & & & & & & & & & & \\
\hline 14 & $1 / 5$ & $1 / 40$ & $\begin{array}{l}\text { L.P.B } \\
+\end{array}$ & & & & & & & & & & & \\
\hline 26 & - & $1 / 80$ & $\begin{array}{l}\text { L.P.B } \\
+\end{array}$ & & & & & & & & & & & \\
\hline 56 & - & $1 / 40$ & $\begin{array}{c}\text { L.P.B } \\
+\end{array}$ & & & & & & & & & & & \\
\hline 23 & $1 / 40$ & $\begin{array}{c}\text { L.P. B } \\
+\end{array}$ & & & & & & & & & & & & \\
\hline 57 & - & $1 / 320$ & $\begin{array}{c}\mathrm{L}+\mathrm{P} \cdot 3 \\
+\end{array}$ & & & & & & & & & & & \\
\hline 15 & $1 / 5$ & $1 / 80$ & $1 / 260$ & L.P.B. & & & & & & & & & & \\
\hline 18 & - & $1 / 80$ & $\begin{array}{c}\overline{\mathrm{L}} \cdot \mathrm{P} \cdot \mathrm{B} \\
+\end{array}$ & & & & & & & & & & & \\
\hline 76 & - & $1 / 80$ & $1 / 160$ & $1 / 320$ & $1 / 160$ & $1 / 160$ & $1 / 80$ & $1 / 40$ & $1 / 40$ & $1 / 40$ & $1 / 20$ & $1 / 20$ & $1 / 20$ & $\begin{array}{l}\text { Gros séquestre péri- } \\
\text { pneumonique }\end{array}$ \\
\hline 95 & $1 / 10$ & $1 / 160$ & $\begin{array}{c}\mathrm{L} . \mathrm{P} . \overline{\mathrm{B}} \\
+\end{array}$ & & & & & & & & & & & \\
\hline
\end{tabular}

$\stackrel{\text { L.․․ }}{+}$ : mort avec lésions péripneumonıques

Tableau 3 - Evolution sérologique des anima.xx intubés du premier lot (Antigène circulant décelé par Frécipito-diffúsion en boste de Pétri)

\begin{tabular}{|c|c|c|c|c|c|c|c|c|c|c|c|c|c|}
\hline $\begin{array}{c}\text { Tombre de jours apres } \\
\text { l'intubation }\end{array}$ & 7 & 13 & 21 & 28 & 35 & 41 & 49 & 56 & 63 & 70 & 77 & 84 & 91 \\
\hline $\begin{array}{c}N^{\circ} \text { des aninaux } \\
13\end{array}$ & - & - & - & - & - & - & - & - & - & - & - & - & - \\
\hline 88 & - & - & + & \begin{tabular}{|c|} 
¿.P.B. \\
+
\end{tabular} & & & & & & & & & \\
\hline 32 & + & $\begin{array}{l}\text { L.P.B. } \\
+\end{array}$ & & & & & & & & & & & \\
\hline 44 & - & + & $\begin{array}{r}\mathrm{L} . \mathrm{P} . \mathrm{B} . \\
+\ldots\end{array}$ & & & & & & & & & & \\
\hline 36 & - & - & - & - & + & + & + & + & + & + & - & - & - \\
\hline 29 & - & + & $\begin{array}{l}\text { L.P.E. } \\
+\end{array}$ & & & & & & & & & & \\
\hline 14 & - & - & $\begin{array}{c}\text { E.P. } \\
+\end{array}$ & & & & & & & & & & \\
\hline 26 & - & - & $\begin{array}{c}\text { I.P.S. } \\
+\end{array}$ & & & & & & & & & & \\
\hline 56 & - & - & $\begin{array}{c}2 . P .3 . \\
+\end{array}$ & & & & & & & & & & \\
\hline 23 & + & $\begin{array}{l}\text { L.P.B. } \\
+\end{array}$ & & & & & & & & & & & \\
\hline 57 & - & - & $\begin{array}{c}\mathrm{L} . \mathrm{P} . \mathrm{B} . \\
+\end{array}$ & & & & & & & & & & \\
\hline 15 & - & - & + & $\begin{array}{c}\text { L.P.B. } \\
+ \\
\end{array}$ & & & & & & & & & \\
\hline 18 & - & - & $\begin{aligned} \\
\quad .9 .8 . \\
\end{aligned}$ & & & & & & & & & & \\
\hline 76 & - & - & - & - & - & - & - & - & - & + & + & + & + \\
\hline 95 & - & - & $\begin{array}{l}\text { L.P. } 3 . \\
\quad+\end{array}$ & & & & & & & & & & \\
\hline
\end{tabular}

L.P.B. : Ilort avec lészon de périp̣neumonie
++

$+\quad$ : Présence d'antigène circulant 
Tableau 4 - Evolution sérojogrque des anımax intubés ău deuxıème lot (Déviation du complément Kolmer)

\begin{tabular}{|c|c|c|c|c|c|c|c|c|c|c|c|c|}
\hline $\begin{array}{l}\text { Jombre de jours } \\
\text { après lntubation }\end{array}$ & 0 & 7 & 10 & 16 & 24 & 31 & 38 & 45 & 52 & 59 & 66 & $\begin{array}{c}\text { Aluattage } \\
84\end{array}$ \\
\hline $\begin{array}{c}\mathrm{N}^{\circ} \text { des anzmaux } \\
702\end{array}$ & - & $1 / 10$ & $1 / 10$ & $1 / 10$ & $1 / 160$ & $\begin{array}{l}\text { L.P. B. } \\
+\end{array}$ & & & & & & \\
\hline 709 & - & $1 / 80$ & $1 / 80$ & $\begin{array}{c}\text { I.P.R. } \\
+\end{array}$ & & & & & & & & \\
\hline $7 \geq 0$ & - & $1 / 5$ & $1 / 20$ & $1 / 80$ & $\begin{array}{c}\text { L.?.B. } \\
+\end{array}$ & & & & & & & \\
\hline 721 & - & $1 / 5$ & $1 / 20$ & $1 / 80$ & $1 / 320$ & 1/16́0 & $1 / 160$ & $\begin{array}{l}\text { L.P.S. } \\
+\end{array}$ & & & & \\
\hline 722 & - & $1 / 5$ & $1 / 10$ & $1 / 20$ & $1 / 80$ & $1 / 160$ & $\begin{array}{l}\text { L.․․․ } \\
+\end{array}$ & & & & & \\
\hline 724 & - & - & $1 / 20$ & $1 / 40$ & $1 / 160$ & $1 / 80$ & $1 / 40$ & $1 / 10$ & $1 / 10$ & $1 / 10$ & $1 / 10$ & $\begin{array}{l}\text { Séquestre péri } \\
\text { pneumonique }\end{array}$ \\
\hline 715 & $1 / 5$ & $1 / 10$ & $1 / 20$ & $1 / 40$ & L.P.T. & & & & & & & \\
\hline 34 & - & $1 / 20$ & $1 / 160$ & $\begin{array}{c}\text { Lu+P.3. } \\
+\end{array}$ & & & & & & & & \\
\hline 717 & - & $1 / 20$ & $1 / 40$ & $\begin{array}{c}\text { L.P.3. } \\
+\end{array}$ & & & & & & & & \\
\hline 716 & - & $1 / 5$ & $1 / 5$ & $1 / 40$ & $\begin{array}{c}\text { I.P.B. } \\
+\end{array}$ & & & & & & & \\
\hline 7 III & - & $1 / 20$ & $1 / 80$ & $\begin{array}{c}\overline{\mathrm{L}} \cdot \mathrm{P} \cdot \overline{\mathrm{B}} . \\
+\end{array}$ & & & & & & & & \\
\hline 718 & - & $1 / 5$ & $1 / 40$ & $1 / 160$ & $\begin{array}{c}\overline{\mathrm{I}} \cdot \mathrm{P} \cdot \overline{\mathrm{B}} \\
+\end{array}$ & & & & & & & \\
\hline
\end{tabular}

L. ב.B. : mort avec lésions pérłpneumoniques

Tableau 5 - Evolution sérologique des animaux intubés du deuxième lot (Antigène circulent décelé par précipito-diffusion en bồte de pétri)

\begin{tabular}{|c|c|c|c|c|c|c|c|c|c|c|c|}
\hline $\begin{array}{l}\text { Nombre de jours } \\
\text { apres intubation }\end{array}$ & 0 & 7 & 10 & 16 & 24 & 31 & 38 & 45 & 52 & 59 & 66 \\
\hline $\begin{array}{c}\mathbb{N}^{\circ} \text { des animaux } \\
702\end{array}$ & - & - & - & + & + & $\begin{array}{l}\text { I. P.B. } \\
+\end{array}$ & & & & & \\
\hline 709 & $\cdots$ & - & + & $\begin{array}{c}\text { I. P. B. } \\
+\end{array}$ & & & & & & & \\
\hline 710 & - & - & - & - & $\begin{array}{c}\text { L.P.B. } \\
+\end{array}$ & & & & & & \\
\hline 721 & - & - & - & - & + & + & + & $\begin{array}{c}\mathrm{L} . \mathrm{P} \cdot \mathrm{B} . \\
+\end{array}$ & & & \\
\hline 722 & - & - & - & - & - & + & $\begin{array}{c}\text { L.P.B. } \\
+\end{array}$ & & & & \\
\hline 724 & - & - & - & - & - & - & - & - & - & - & - \\
\hline 715 & - & - & - & + & $\begin{array}{c}\mathrm{L} . \mathrm{P} . \mathrm{B} . \\
+\end{array}$ & & & & & & \\
\hline 34 & - & - & - & $\begin{array}{c}\text { I.P.B. } \\
+\end{array}$ & & & & & & & \\
\hline 717 & - & - & - & $\begin{array}{c}\mathrm{L} . \mathrm{P} . \mathrm{B} . \\
+\end{array}$ & & & & & & & \\
\hline 716 & - & - & - & + & $\begin{array}{c}\mathrm{I} * \mathrm{P} . \mathrm{B} . \\
+\end{array}$ & & & & & & \\
\hline 711 & - & - & + & $\begin{array}{c}\text { L.P.B. } \\
+\end{array}$ & & & & & & & \\
\hline 718 & - & - & - & + & $\begin{array}{c}\text { L.P.B. } \\
+ \\
\end{array}$ & & & & & & \\
\hline
\end{tabular}

I.P.B. : mort avec lésions péripneumoniques 


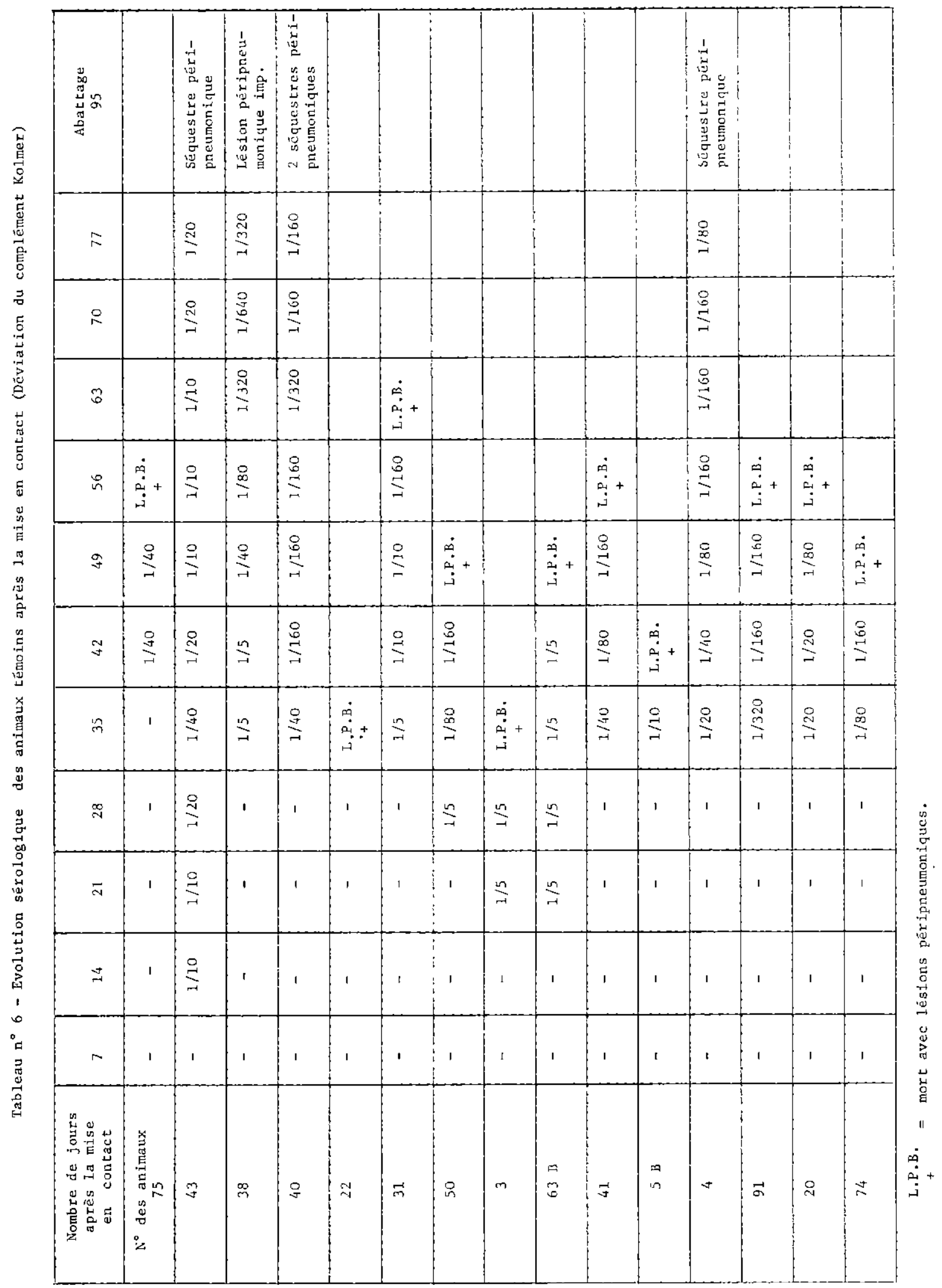


Tableau 7 - Evolution sérologique des animaux témoins après la mise en contact (Antigène circulant décelé par précipito-dif ̃usion en bôte de Pétri)

\begin{tabular}{|c|c|c|c|c|c|c|c|c|c|c|c|}
\hline $\begin{array}{l}\text { Nombre de jours } \\
\text { après la mise } \\
\text { en contact } \\
\end{array}$ & 7 & 14 & 21 & 28 & 35 & 42 & 49 & 56 & 63 & 70 & 77 \\
\hline $\begin{array}{c}\mathrm{N}^{0} \text { des animaux } \\
75\end{array}$ & - & $=$ & $\therefore$ & - & $=$ & $=$ & - & Mort & & & \\
\hline 43 & - & - & - & - & - & - & - & - & - & - & - \\
\hline 38 & - & - & - & - & - & - & - & + & + & + & + \\
\hline 40 & - & - & - & - & - & - & - & - & - & - & - \\
\hline 22 & - & - & - & + & Mort & & & & & & \\
\hline 31 & - & - & - & - & - & - & + & + & Mort & & \\
\hline 50 & - & - & - & - & - & + & Mort & & & & \\
\hline 3 & - & - & - & - & Mort & & & & & & \\
\hline $63 \mathrm{~B}$ & - & - & - & - & - & - & Mort & & & & \\
\hline 41 & - & - & - & - & - & - & - & Mort & & & \\
\hline $5 \mathrm{~B}$ & - & - & - & - & - & Mort & & & & & \\
\hline 4 & - & - & - & - & - & - & + & + & + & + & + \\
\hline 91 & - & - & - & - & - & - & - & Mort & & & \\
\hline 20 & - & - & - & - & - & + & + & Mort & & & \\
\hline 74 & - & - & - & - & - & - & Mort & & & & \\
\hline
\end{tabular}

Tableau 8 - Evolution sérologique des animauy vaccinés après la mise en contact (Déviation du complément Kolmer)

\begin{tabular}{|c|c|c|c|c|c|c|c|c|c|c|c|c|c|}
\hline $\begin{array}{l}\text { Nonbre de jours } \\
\text { après la mise } \\
\text { en contact }\end{array}$ & 0 & 7 & 14 & 21 & 27 & 35 & 42 & 49 & 56 & 63 & 70 & 77 & $\begin{array}{c}\text { Abatt,age } \\
95\end{array}$ \\
\hline $\begin{array}{c}\text { No }^{\circ} \text { des anirawx } \\
25\end{array}$ & - & $1 / 10$ & $1 / 10$ & $1 / 20$ & $1 / 10$ & $1 / 20$ & $1 / 10$ & $1 / 10$ & - & - & - & $=$ & P.I. \\
\hline 24 & - & - & - & - & $1 / 5$ & $1 / 5$ & $1 / 5$ & $=$ & - & $=$ & - & - & P.I. \\
\hline 9 & - & - & - & - & $\begin{array}{l}\text { Nort sans } \\
\text { Iésion P. B }\end{array}$ & & & & & & & & \\
\hline 42 & $1 / 5$ & $1 / 5$ & $1 / 5$ & $2 / 5$ & - $-2-2-1$ & - & $\begin{array}{l}\text { Mort sans } \\
\text { lésion Pra }\end{array}$ & & & & & & \\
\hline 83 & - & $1 / 5$ & $1 / 5$ & $2 / 10$ & $1 / 80$ & $1 / 80$ & $1 / 40$ & $1 / 20$ & $1 / 10$ & $1 / 5$ & $1 / 5$ & $1 / 10$ & P.I. \\
\hline 10 & - & - & - & - & $2 / 5$ & $1 / 5$ & $1 / 5$ & $\therefore 1 / 10$ & - & - & - & $1 / 5$ & P.I. \\
\hline 79 & - & - & - & - & - & $1 / 10$ & $1 / 10$ & $2 / 20$ & - & - & $1 / 5$ & $1 / 10$ & P.I. \\
\hline $11 \mathrm{~B}$ & - & $1 / 5$ & $1 / 5$ & $1 / 5$ & $1 / 5$ & $1 / 5$ & - & - & - & - & - & - & P.I. \\
\hline 80 & - & - & $1 / 5$ & $1 / 5$ & $1 / 5$ & $1 / 5$ & $1 / 5$ & $1 / 5$ & - & - & - & - & P.I. \\
\hline 90 & $1 / 5$ & $1 / 20$ & $1 / 20$ & $1 / 20$ & $1 / 10$ & $1 / 10$ & $1 / 5$ & $1 / 5$ & - & - & - & - & P.I. \\
\hline 11 & - & - & $I / 5$ & $1 / 5$ & $1 / 5$ & $1 / 5$ & - & - & - & - & - & - & Э.I. \\
\hline 82 & - & - & - & - & - & $1 / 5$ & - & - & - & - & $1 / 5$ & $1 / 5$ & P.I. \\
\hline 2 & $-\quad \cdots$ & $\because-$ & - & - & - & $=$ & - & $1 / 5$ & - & - & 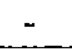 & $=$ & P.I. \\
\hline $18 \mathrm{~B}$ & - & - & - & - & $1 / 5$ & $1 / 40^{\circ}$ & $1 / 40$ & $1 / 80$ & $1 / 40$ & $1 / 20$ & $1 / 20$ & $1 / 20$ & P.I. \\
\hline 93 & - & - & - & - & - & - & - & $\begin{array}{l}\text { Mort sans } \\
\text { lésion ?.B }\end{array}$ & & & & & \\
\hline
\end{tabular}


qui suivirent la mise en contact. Les 4 survivants ont été sacrifiés au $95^{\circ}$ jour; trois (43, 38 et 40 ) montraient des séquestres encapsulés, un (38) une lésion évolutive englobant la totalité d'un lobe pulmonaire.

\section{c) Evolution des animaux vaccinés}

L'évolution sérologique (déviation du complément) fait l'objet du tableau 8 .

Sur 15 animaux vaccinés, trois $(9,42,93)$ ont succombé en cours d'expérience sans que l'examen post-mortem ne révèle la moindre lésion pulmonaire. La mort de ces animaux peut s'expliquer par le fait que, dans les conditions difficiles imposées par le confinement, des différences de taille et de vitalité empêchent certains sujets d'atteindre les mangcoires et de s'alimenter normalement. La cachexie s'installe et la mort survient par épuisement.

Les 12 bovins vaccinés survivants ont été sacrifiés 95 jours après la mise en contact avec les animaux infectants. Aucun d'eux ne présentait la moindre atteinte pulmonaire et $M$. mycoides n'a pu être isolé d'aucun ganglion trachéo-bronchique.

Sur le plan sérologique, la presque totalité des animaux vaccinés a présenté une montée d'anticorps fixant le complément au cours du contact avec les animaux infectés expérimentalement et les témoins en cours d'infection. Cette évolution traduit l'existence d'un stimulus antigénique certain qui ne va pas, grâce à l'immunité existante, jusqu'au développenent de lésion pulmonaire.

\section{CONCLUSION}

A la suite d'une expérience contact réaliséc trois mois après la vaccination avec 15 bovins vaccinés à l'aide d’un vaccin lyophilisé préparé avec la souche $T_{1}$ (44 passage), trois bovins ont succombé pour des raisons étrangères à la péripneumonie bovine; les douze survivants, sacrifiés 95 jours après le début de la mise en contact, on présenté à l'autopsie un appareil pulmonaire indemne de toute atteinte péripneumonique. Ce résultat a été observé à la fois chez des animaux qui avaient offert une réaction locale post-vaccinale au point d'inoculation et chez des individus où aucune réaction locale n'avait été enregistrée. Ainsi, trois mois après la vaccination avec le vaccin $T_{1}$ lyophilisé, l'immunité conférée est totale.

\section{B. VALEUR DE L'IMMUNITE SEPT MOIS ET DEMI APRES LA VACCINATION}

La seconde phase de l'expérience débute en mars 1969. Lors de la mise en contact, les animaux immunisés, éprouvés, ont été vaccinés depuis 7 mois et demi.

\section{Intubation des animaux destinés à devenir infectants}

L'opération est effectuée comme précédemment. Elle porte sur 15 bovins : $\mathrm{n}^{\text {os }}$ I 170, I 171, I 167, I 165, I 162, I 189, I 174, I 169, I 175, I 160, I 177, I 161, I 164, I 188, I 196.

L'inoculum est préparé à partir de lésions péripneumoniques recueillies sur un animal malade, abattu sur le terrain une semaine plus tôt.

\section{Mise en contact étroit des animaux vaccinés, des témoins non vaccinés et des intubés}

Quinze bovins vaccinés sont prélevés dans le troupeau immunisé. Ce sont les $\mathrm{n}^{\circ \mathrm{v}} \mathrm{V} 255 \mathrm{R}$, V 66 B, V 75 B, V 69 B, V 19 B, V 32 B, V $80 \mathrm{~B}, \mathrm{~V} 39, \mathrm{~V} 253 \mathrm{R}, \mathrm{V} 99 \mathrm{~B}, \mathrm{~V} 17 \mathrm{~B}$, V $254 \mathrm{R}, \mathrm{V} 64, \mathrm{~V} 43 \mathrm{~B}$.

Les 15 animaux témoins portent les $\mathrm{n}^{\mathrm{0s}}$ : $\mathrm{T} 183$, T 168, T 139, T 199, T 179, T 181, T 186, T 190, T 187, T 185, T 149, T 166, T 163, T 1158 et $\mathrm{T} 176$.

\section{Evolution des animaux}

Les observations s'arrêtent 95 jours après le début de la mise en contact (abattage).

\section{a) Evolution des animaux infectants}

L'évolution sérologique (déviation du complément Kolmer) est rapportée dans le tableau 9.

Sur les 15 animaux infectés expérimentalement. 8 ont succombé en présentant des lésions typiques de péripneumonie entre le $22^{\mathrm{e}}$ et le $64^{\mathrm{e}}$ jour à compter du jour de l'intubation. Les 


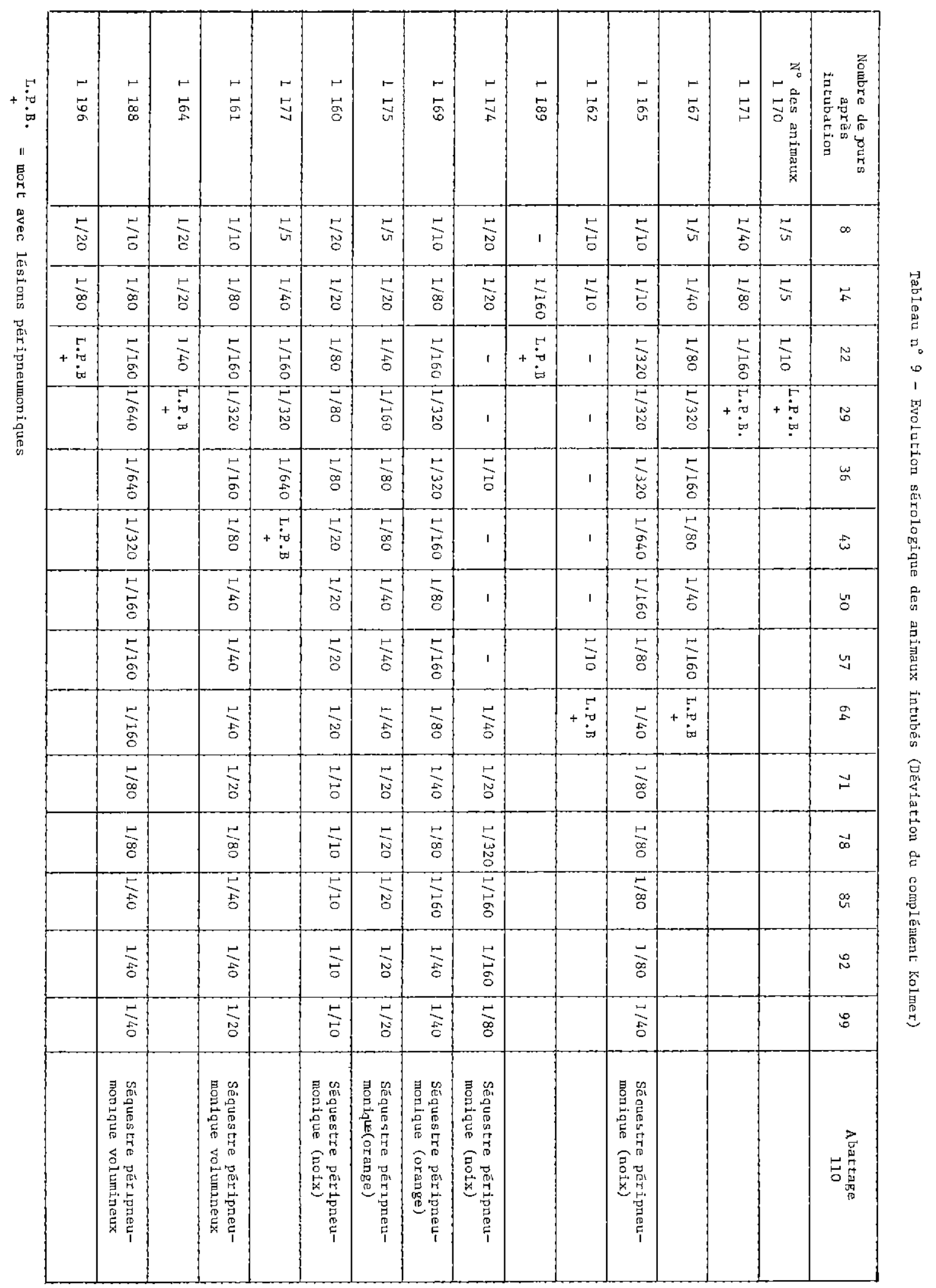


7 autres, abattus 110 jours après l'infection endobronchique, offraient des séquestres pulmonaires de taille variable (tableau 9).

\section{b) Evolution des témoins}

L'évolution sérologique de ces animaux fait l'objet du tableau 10.

Sur 15 témoins, 10 ont succombé en montrant à l'autopsie des lésions typiques de péri- pneumonie. Les morts s'étalent entre le $28^{\mathrm{e}}$ et le $84^{\mathrm{e}}$ jour suivant le début de la mise en contact; un maximum est observé autour du $56^{\mathrm{e}}$ jour.

Les 5 témoins survivants sont sacrifiés 95 jours après le début de l'expérience: un présente un poumon indemne (T. 168), les quatre autres des séquestres péripneumoniques de taille variable (tableau 10 ).

Tableau 10 - Evolutıon sérologique des animaux témoins après la mise en contact

(Déviation du complément Kolmer)

\begin{tabular}{|c|c|c|c|c|c|c|c|c|c|c|c|c|c|}
\hline $\begin{array}{l}\text { Nombre de jours après } \\
\text { la mase en contact }\end{array}$ & 7 & 14 & 21 & 28 & 35 & 42 & 49 & 56 & 63 & 70 & 77 & 82 & $\begin{array}{l}\text { Whattage } \\
95\end{array}$ \\
\hline $\begin{array}{c}N^{\circ} \text { des animaux } \\
\text { T } 183\end{array}$ & - & - & - & - & - & - & $1 / 40$ & $1 / 40$ & $1 / 40$ & $1 / 40$ & $1 / 40$ & $1 / 20$ & $\begin{array}{l}\text { Séquestre péripneu- } \\
\text { monique (noix) }\end{array}$ \\
\hline T 168 & - & - & - & $1 / 5$ & - & $1 / 5$ & $1 / 10$ & - & $1 / 10$ & - & $1 / 10$ & - & Absence de lésıons \\
\hline T 139 & - & - & - & - & - & - & $1 / 20$ & $1 / 80$ & $1 / 160$ & $1 / 80$ & $1 / 80$ & $1 / 20$ & $\begin{array}{l}\text { Séquestre péripneu- } \\
\text { ronique volumineux }\end{array}$ \\
\hline T 199 & - & - & - & - & - & $1 / 10$ & $1 / 5$ & - & $1 / 40$ & $1 / 160$ & $1 / 160$ & $\begin{array}{c}\mathrm{L.P} \cdot \mathrm{B} \\
+\end{array}$ & \\
\hline 도 179 & - & - & - & - & - & - & $1 / 40$ & $\begin{array}{c}\text { L.P.B } \\
+\end{array}$ & & & & & \\
\hline T 181 & - & - & - & - & - & $1 / 5$ & $1 / 20$ & $1 / 80$ & $1 / 160$ & $1 / 160$ & $1 / 160$ & $1 / 40$ & $\begin{array}{l}\text { Séquestre péripneu- } \\
\text { monioue (noix) }\end{array}$ \\
\hline T 186 & - & - & $1 / 5$ & $1 / 20$ & $1 / 40$ & $\begin{array}{c}\text { L.P.B } \\
+\end{array}$ & & & & & & & \\
\hline T 190 & - & - & - & - & - & - & - & $1 / 40$ & $1 / 160$ & $\begin{array}{c}\text { I.P.B } \\
+\end{array}$ & & & \\
\hline T 187 & - & - & - & - & $1 / 10$ & $1 / 1,0$ & $1 / 40$ & $1 / 40$ & $1 / 40$ & $1 / 40$ & $1 / 40$ & $1 / 20$ & $\begin{array}{l}\text { Séquestre péripneu- } \\
\text { monique (orance) }\end{array}$ \\
\hline T 185 & - & - & - & - & - & - & $1 / 10$ & $\begin{array}{c}\text { I.P.B } \\
+\end{array}$ & & & & & \\
\hline$T 149$ & - & - & - & - & $I / 20$ & $\begin{array}{r}\mathrm{L}, ? . \mathrm{B} \\
+\end{array}$ & & & & & & & \\
\hline T 166 & - & - & $1 / 5$ & - & - & $1 / 10$ & $1 / 40$ & $\begin{array}{c}\bar{L} \cdot \bar{P} \cdot \mathrm{B} \\
+\end{array}$ & & & & & \\
\hline T 163 & - & - & - & - & - & $1 / 5$ & $1 / 40$ & $\begin{array}{c}\text { I.P.B } \\
+\end{array}$ & & & & & \\
\hline T $115 \mathrm{~S}$ & - & - & $1 / 20$ & $\begin{array}{c}\text { S.L.P.3 } \\
+\end{array}$ & & & & & & & & & \\
\hline T 176 & - & - & $1 / 5$ & - & - & $1 / 5$ & $1 / 40$ & $1 / 160$ & $1 / 160$ & $\begin{array}{c}\mathrm{L} . \mathrm{P} . \mathrm{B} \\
+\end{array}$ & & & \\
\hline
\end{tabular}

$$
\begin{aligned}
& \begin{array}{l}
\text { L.P.B. } \\
+
\end{array} \\
& \begin{array}{l}
\text { S.L.P.B isort crec lésions péripreumoniq.tes } \\
+
\end{array} \text { : iort sans lésion péripneumonique }
\end{aligned}
$$

\section{c) Evolution des animaux vaccinés}

Les 15 bovins vaccinés ont survécu aux conditions sévères du confinement. Abattus 95 jours après le commencement de l'épreuve, ils offrent à l'autopsie un appareil pulmonaire indemne de toute atteinte pulmonaire et $M$. mycoides n'est isolé d'aucun ganglion trachéo-bronchique. L'évolution sérologique figure dans le tableau 11.

\section{CONCLUSION}

Sept mois et demi après la vaccination à l'aide du vaccin lyophilisé $T_{1}$, quinze bovins mis en contact pendant 95 jours avec des animaux infectants ont présenté après abattage un appareil pulmonaire intact. Au bout de ce temps, l'immunité conférée par la vaccination est totale. 
Tableav 11 - Evolution sérologique des animaux vaccinés après la mise en contact (Dévietion du complément' Kolner)

\begin{tabular}{|c|c|c|c|c|c|c|c|c|c|c|c|c|c|}
\hline $\begin{array}{l}\text { Nombre de jours apres } \\
\text { la mise en contact }\end{array}$ & 7 & 14 & 21 & 28 & 35 & 42 & 49 & 56 & 63 & 70 & 77 & 84 & $\begin{array}{c}\text { Abattage } \\
95\end{array}$ \\
\hline $\begin{array}{l}\mathrm{H}^{\circ} \text { des animaux } \\
\mathrm{V} 255 \mathrm{R}\end{array}$ & - & - & - & - & - & - & - & - & $1 / 5$ & - & $1 / 10$ & - & P.I. \\
\hline V $66 \mathrm{~B}$ & - & - & - & - & - & - & - & - & - & - & - & - & P.I. \\
\hline $\mathrm{V} 75 \mathrm{~B}$ & - & - & - & - & - & - & $2 / 10$ & - & - & - & - & - & P.I. \\
\hline $\mathrm{V} \quad 69 \mathrm{~B}$ & - & - & $1 / 5$ & - & - & - & - & - & $1 / 10$ & - & - & - & P.'. \\
\hline $\mathrm{V} \quad 19 \mathrm{~B}$ & - & - & - & - & - & - & - & - & $1 / 10$ & - & - & - & P.I. \\
\hline $\mathrm{V} \quad 32 \mathrm{~B}$ & - & - & $1 / 10$ & - & - & - & - & - & - & - & - & - & P.I. \\
\hline $\mathrm{V} \quad 50 \mathrm{~B}$ & - & - & - & - & - & $I / 20$ & $1 / 5$ & - & $1 / 5$ & - & - & - & P.I. \\
\hline V 39 & - & - & $1 / 5$ & - & - & - & - & - & $1 / 10$ & - & $1 / 5$ & - & P.I. \\
\hline$V 253 \mathrm{R}$ & - & - & - & - & - & - & - & - & $2 / 5$ & - & - & - & P.I. \\
\hline $\mathrm{V} 99 \mathrm{~B}$ & - & - & - & - & - & - & $1 / 5$ & - & - & $1 / 10$ & - & - & P.I. \\
\hline $\mathrm{V} 17 \mathrm{~B}$ & - & - & - & - & - & - & - & - & $1 / 5$ & - & $1 / 5$ & - & P.I. \\
\hline $\mathrm{V} 254 \mathrm{R}$ & - & - & - & - & - & - & $1 / 5$ & - & - & - & $1 / 5$. & - & P.I. \\
\hline$v 64$ & $1 / 10$ & $1 / 5$ & $I / 10$ & $1 / 5$ & - & - & $1 / 5$ & - & $1 / 10$ & $1 / 10$ & $1 / 10$ & - & D.I. \\
\hline $\mathrm{V} \quad 94$ & - & $1 / 10$ & $1 / 5$ & - & - & - & - & - & - & - & - & - & P.I. \\
\hline $\mathrm{V} 43 \mathrm{~B}$ & - & - & - & - & - & - & - & - & $1 / 10$ & - & - & - & P.I. \\
\hline
\end{tabular}

P.I. : Poumon indemne

\section{VALEUR DE L'IMMUNITE QUATORZE MOIS APRES LA VACCINATION}

La troisième phase de l'expérience débute en septembre 1969. Lors de la mise en contact, les animaux immunisés, éprouvés, ont été vaccinés depuis 14 mois. Il était prévu. à l'origine, d'effectucr l'épreuve deux mois plus tôt (test d'immunité un an après la vaccination). La difficulté de se procurer des animaux pendant la saison des pluies est responsable du changement apporté au protocole.

\section{Intubation des animaux destinés à devenir infectants}

L'inoculum est préparé à partir des lésions péripneumoniques recueillies sur un animal malade, abattu en brousse (Nioro du Rip) une semaine avant l'opération.

Cette dernière porte sur 15 bovins : $\mathrm{n}^{\mathrm{0s}} \mathbf{I} 08$, I 87, I 41, I 57, I 45, I 73, I 47, I 31, I 24 , I 58, I 97, I 85, I 22, I 40, I 51.

\section{Mise en contact étroit des animaux vaccinés, des témoins non vaccinés et des intubés}

Les 10 bovins restants du troupeau immunisé sont utilisés (à l'origine 50 bovins avaient été vaccinés, 30 ont été prélevés pour les deux premières expériences, 10 ont donc disparu pendant les 14 mois d'entretien pour des raisons diverses). Ce sont les $\mathrm{n}^{\text {ns }} \mathrm{V} 519, \mathrm{~V} 512$, V 509, V 533, V 38 B, V 513, V 581, V 517 , V 582, V 595.

Les dix animaux témoins portent les $\mathrm{N}^{\mathrm{as}}$ : T 509, T 520, T 526, T 516, T 532, T 518, T 528, T 504, T 523, T 527.

\section{Evolution des animaux}

Les observations s'arrêtent 96 jours après le début de la mise en contact (abattage).

\section{a) Evolution des animaux infectants}

L'évolution sérologique est rapportée dans les tableaux 12 (déviation du complément Kolmer) et 13 (antigène circulant). Des précipitines 
Tableau 12 - Evolutior sérologique des animaux intubés (Déviation du complément Kolmer)

\begin{tabular}{|c|c|c|c|c|c|c|c|c|c|c|c|c|}
\hline $\begin{array}{l}\text { Nombre de jours } \\
\text { après intubation }\end{array}$ & 7 & 24 & 21 & 28 & 35 & 42 & 49 & 56 & 63 & 70 & 77 & 84 \\
\hline $\begin{array}{c}N^{\circ} \text { des anzmaux } \\
\text { I } 08\end{array}$ & - & $1 / 10$ & 1/160 & $\begin{array}{c}\text { L.?. } \\
+\end{array}$ & & & & & & & & \\
\hline I 87 & - & $1 / 10$ & $1 / 160$ & $1 / 80$ & $1 / 80$ & $1 / 10$ & $1 / 10$ & $\begin{array}{c}\text { S.P. } \\
+\end{array}$ & & & & \\
\hline I 41 & - & $1 / 5$ & $1 / 320$ & $1 / 640$ & $1 / 640$ & $1 / 320$ & $1 / 40$ & $1 / 20$ & L.P. & & & \\
\hline I 57 & - & $1 / 5$ & $1 / 160$ & $\begin{array}{c}\mathrm{L} \cdot \overline{\mathrm{F}} \cdot \overline{\mathrm{B}} \\
+\end{array}$ & & & & & & & & \\
\hline$I 45$ & - & $1 / 10$ & $1 / 10$ & $1 / 80$ & $1 / 160$ & $1 / 160$ & $1 / 160$ & $\begin{array}{c}\mathrm{I} \cdot \mathrm{P} \cdot \mathrm{B} \\
+\end{array}$ & & & & \\
\hline I 73 & - & $2 / 5$ & $1 / 40$ & $\begin{array}{c}\text { L.P.B } \\
+\end{array}$ & & & & & & & & \\
\hline I 47 & - & $1 / 10$ & $1 / 160$ & $\begin{array}{l}\text { L.P.B } \\
+\end{array}$ & & & & & & & & \\
\hline I 31 & $1 / 5$ & $1 / 10$ & $1 / 320$ & $1 / 320$ & $\begin{array}{c}\text { L. P.3 } \\
+\end{array}$ & & & & & & & \\
\hline I 24 & $1 / 5$ & $1 / 5$ & $1 / 10$ & $1 / 320$ & $1 / 320$ & $1 / 640$ & $1 / 640$ & $\begin{array}{c}\mathrm{I} \cdot \overrightarrow{\mathrm{P} \cdot \mathrm{B}} \\
+\end{array}$ & & & & \\
\hline I 58 & $1 / 5$ & $1 / 10$ & $1 / 10$ & $1 / 10$ & $1 / 20$ & $1 / 80$ & $1 / 64 \mathrm{C}$ & $1 / 320$ & $\begin{array}{c}\mathrm{L} . \bar{P} \cdot \overline{\mathrm{B}} \\
+\end{array}$ & & & \\
\hline I 97 & $1 / 5$ & $1 / 5$ & $1 / 20$ & $1 / 20$ & $1 / 40$ & $1 / 80$ & $1 / 40$ & $1 / 10$ & $1 / 10$ & $1 / 10$ & $1 / 10$ & $\begin{array}{c}5 . \mathrm{P} \\
+\end{array}$ \\
\hline 185 & $1 / 5$ & $1 / 5$ & $1 / 5$ & $1 / 40$ & $1 / 80$ & $\begin{array}{c}\text { L.P.3 } \\
+\end{array}$ & & & & & & \\
\hline I 22 & - & $1 / 20$ & $\begin{array}{l}\mathrm{C} . \mathrm{P} . \\
+ \\
\end{array}$ & & & & & & & & & \\
\hline$I 40$ & $1 / 5$ & $\begin{array}{l}\text { S.P. } \\
+ \\
\end{array}$ & & & & & & & & & & \\
\hline I 51 & - & $\begin{array}{c}\mathrm{C.P} \\
+\end{array}$ & & & & & & & & & & \\
\hline
\end{tabular}

c.P. . Mort, congestion pulmonaire

S.P. : wort, séquestre pérıpoumonique

L.P.13. : Mom, lêsıon férigneunonique

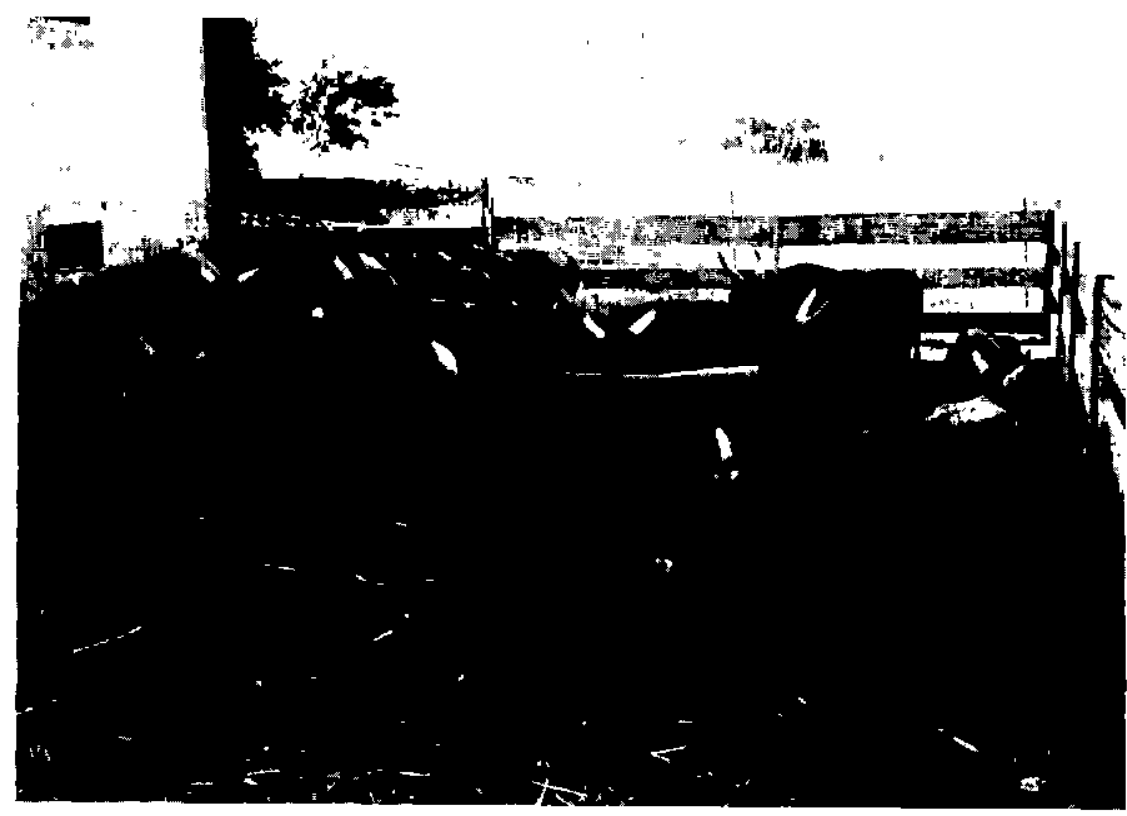

Etat du troupeau d'expérience ( $3^{\mathrm{a}}$ phase : immunité 14 mols) 56 jours après le début de la mise en contact. A cette date, il restait un bovin infectant, 5 témoins et les 10 bovins vaccinés. 
Tableau 13 - Evolution sérologique des animaux intubés

(Antigònc circulant aécelé par précîpizo-diffusion en boite de Pétri)

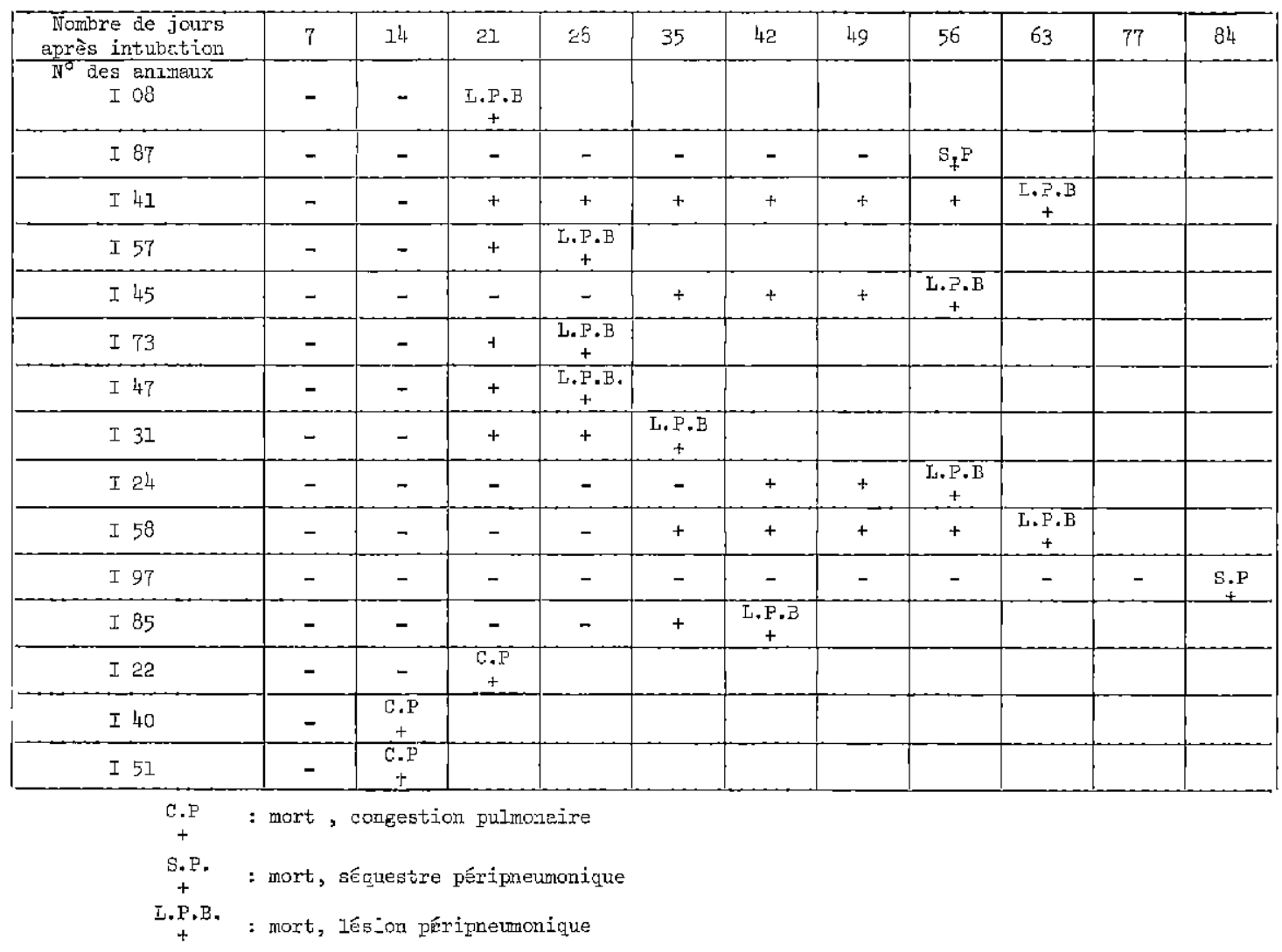

Tảoleau 1' - Itolution sérologique des animaux témoins après la mise en contact (Déviation du complément Kolmer)

\begin{tabular}{|c|c|c|c|c|c|c|c|c|c|c|c|c|c|c|}
\hline $\begin{array}{l}\text { Nombre de jours } \\
\text { après la mise en } \\
\text { contact }\end{array}$ & 7 & 14 & 21 & 28 & 35 & 42 & 49 & 56 & 63 & 70 & 77 & 83 & 91 & $\begin{array}{c}\text { Abst tage } \\
96\end{array}$ \\
\hline $\begin{array}{r}\mathrm{N}^{\circ} \text { des animaux } \\
\text { T } 509\end{array}$ & $1 / 5$ & $1 / 5$ & $1 / 5$ & $1 / 10$ & $\begin{array}{c}\mathrm{L} \cdot \mathrm{P}, \mathrm{B} \\
+ \\
\end{array}$ & & & & & & & & & \\
\hline Т 520 & $1 / 5$ & $1 / 5$. & - & - & - & - & $\infty$ & $1 / 5$ & $I / 20$ & $1 / 320$ & $\begin{array}{c}\mathrm{L} \cdot \mathrm{P} \cdot \mathrm{B} \\
+\end{array}$ & & & \\
\hline T 526 & $1 / 5$ & $1 / 5$ & $1 / 5$ & - & - & $1 / 80$ & $1 / 320$ & $1 / 80$ & $\begin{array}{c}\overrightarrow{I . P . B} \\
+\end{array}$ & & & & & \\
\hline T 5.16 & $1 / 5$ & $1 / 5$ & $1 / 5$ & - & - & $1 / 40$ & $1 / 160$ & $\begin{array}{c}\text { L.P.B } \\
+\end{array}$ & & & & & & \\
\hline T 532 & $1 / 5$ & $1 / 5$ & $1 / 5$ & $1 / 10$ & $1 / 10$ & $1 / 40$ & $\begin{array}{c}\mathrm{I} \cdot \mathrm{P} \cdot \mathrm{B} \\
+\end{array}$ & & & & & & & \\
\hline T 518 & $1 / 5$ & - & - & - & - & $1 / 10$ & $1 / 40$ & $1 / 40$ & $1 / 160$ & $1 / 80$ & $1 / 40$ & $1 / 40$ & $1 / 40$ & $\begin{array}{l}\text { Séquestre } \\
\text { (orange) }\end{array}$ \\
\hline T 528 & - & $1 / 5$ & - & - & $\begin{array}{c}\text { L.P.B } \\
+\end{array}$ & & & & & & & & & \\
\hline T 504 & $1 / 10$ & $1 / 10$ & $\begin{array}{r}\mathrm{L} \cdot \mathrm{P} \cdot \mathrm{B} \\
+ \\
\end{array}$ & & & & & & & & & & & \\
\hline T 523 & - & $1 / 5$ & $1 / 5$ & - & - & - & $1 / 5$ & $1 / 40$ & $1 / 160$ & $\begin{array}{c}\mathrm{L.P.B} \\
+\end{array}$ & & & & \\
\hline T 527 & $1 / 5$ & - & $1 / 5$ & - & - & - & - & - & $1 / 10$ & $1 / 5$ & $1 / 5$ & $\begin{array}{c}\mathrm{L.P} \cdot \mathrm{B} \\
+\end{array}$ & & \\
\hline
\end{tabular}

L.P.B. : Mort avec lésions péripneumoniques
+ 


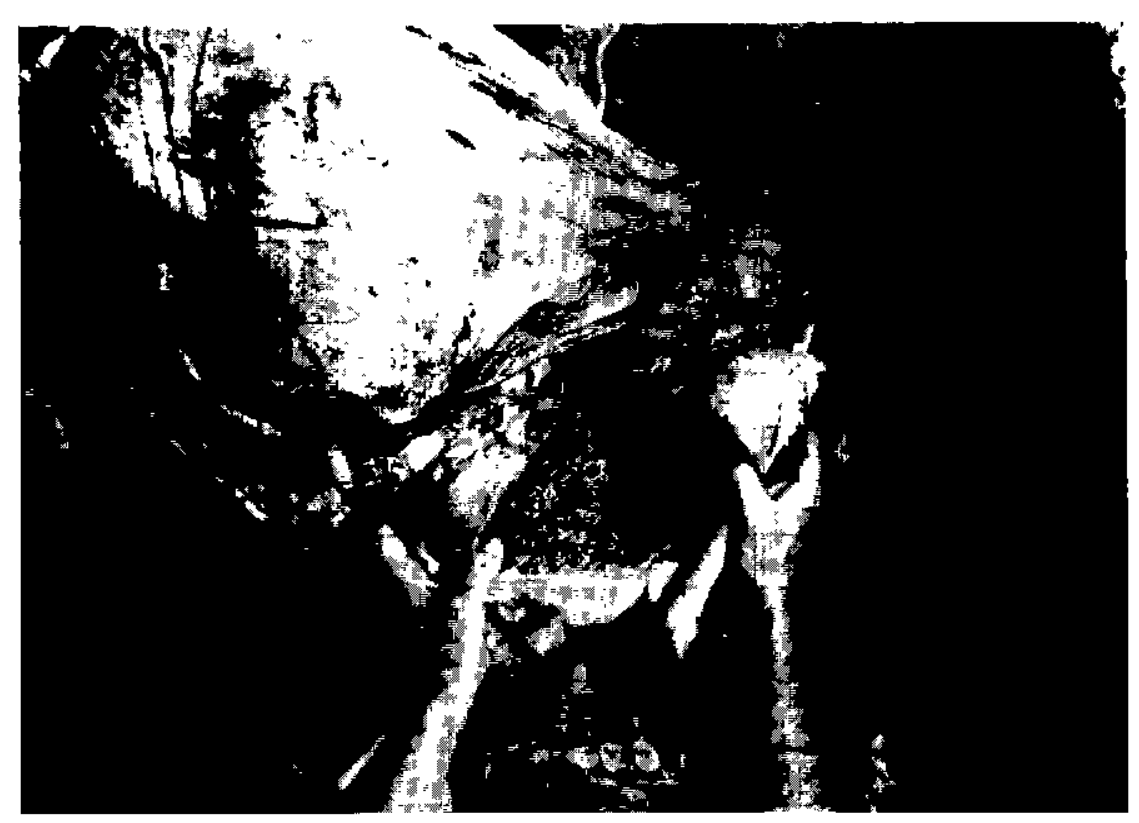

Lésions pulmonaires d'un témoin mort de péripneumonie.

(antigalactane) ont été décelées chez le T 24, 28 jours après l'intubation. Aucun des bovins infectés expérimentalement n'a atteint le terme de l'expérience. La mort des animaux ayant présenté des lésions péripneumoniques caractéristiques se situe entre le $28^{e}$ et le $63^{r}$ jour suivant l'intubation. Cette évolution recoupe les observations effectuées lors de la deuxième expérience.

\section{b) Evolution des témoins}

L'évolution sérologique est rapportée dans les tableaux 14 (déviation du complément Kolmer) et 15 (antigène circulant). Sur 10 témoins, 9 ont succombé en montrant à l'autopsie des lésions caractéristiques (photo 2). Les morts s'étalent entre le $21^{\circ}$ et le $77^{\circ}$ jour suivant la mise en contact. Un bovin qui fixe le complément au 1/160, 63 jours après le début de

Tableau 15 - Evolution sérologique des animaux témoins après la mise en contact (antigène crrculant décelé par précipito-diffusion en boste de Pétri)

\begin{tabular}{|c|c|c|c|c|c|c|c|c|c|c|c|c|c|c|}
\hline $\begin{array}{l}\text { Nombre de jours } \\
\text { après la mise en } \\
\text { contact }\end{array}$ & 7 & 14 & 21 & 20 & 35 & 42 & 49 & 56 & 63 & 70 & 77 & 83 & 91 & $\begin{array}{c}\text { Abattage } \\
96\end{array}$ \\
\hline $\begin{array}{c}\mathbb{N}^{\circ} \text { des animeux } \\
\mathrm{T} 509\end{array}$ & - & - & - & - & $\begin{array}{c}L \cdot P \cdot B \\
+\end{array}$ & & & & & & & & & \\
\hline T 520 & - & - & - & - & - & - & - & - & - & + & $\begin{array}{c}\mathrm{L} . \mathrm{P} . \mathrm{B} \\
+\end{array}$ & & & \\
\hline T 526 & - & - & - & - & - & + & + & + & $\begin{array}{c}\text { L.P.B } \\
+\end{array}$ & & & & & \\
\hline T 516 & $=$ & - & - & - & - & - & - & $\begin{array}{c}\mathrm{L} . \mathrm{P}, \mathrm{B} \\
+ \\
\end{array}$ & & & & & & \\
\hline T 532 & - & - & - & - & - & - & $\begin{array}{c}\text { L. } \\
+.3 \\
\end{array}$ & & & & & & & \\
\hline T 518 & - & - & - & - & - & - & + & + & + & + & + & + & + & \\
\hline T 528 & - & - & - & - & $\begin{array}{c}\text { L. } \begin{array}{c}\text { L. } 3 \\
+\end{array} \\
\end{array}$ & & & & & & & & & \\
\hline T 504 & - & - & $\begin{array}{c}\text { L.․․․ } \\
+\end{array}$ & & & & & & & & & & & \\
\hline T 523 & - & - & - & - & - & - & - & - & $T$ & $\begin{array}{c}\bar{L} . \mathrm{F} . \mathrm{B} \\
+\end{array}$ & & & & \\
\hline T 527 & - & - & - & - & - & - & - & - & - & - & - & $\begin{array}{c}\text { L.P.B } \\
+\end{array}$ & & \\
\hline
\end{tabular}

L.P.B. : mort avec lésions péripneumoniques
+ 
Tableau 16 - Bvolution sérologique des animaux vaccinés après la mise en contact (déviation du complément Kolrer)

\begin{tabular}{|c|c|c|c|c|c|c|c|c|c|c|c|c|c|c|}
\hline $\begin{array}{l}\text { Nombre de jours } \\
\text { après la mise en } \\
\text { contact }\end{array}$ & 7 & 14 & 21 & 28 & 35 & 42 & 49 & 56 & 63 & 70 & 77 & 83 & 91 & $\begin{array}{c}\text { Abattage } \\
96\end{array}$ \\
\hline $\begin{array}{c}\mathbb{N}^{9} \text { des animaux } \\
\text { V } 519\end{array}$ & $1 / 5$ & $1 / 5$ & $1 / 5$ & - & $1 / 5$ & - & - & - & $1 / 5$ & $1 / 5$ & - & $1 / 5$ & - & P.I. \\
\hline$v 512$ & $1 / 5$ & $1 / 5$ & $1 / 5$ & - & $1 / 5$ & $1 / 5$ & $1 / 5$ & - & - & $1 / 5$ & - & - & - & P.I. \\
\hline$\forall 509$ & $1 / 5$ & $1 / 5$ & - & - & $1 / 5$ & - & - & - & $1 / 5$ & $1 / 5$ & - & - & - & P.I. \\
\hline V 533 & $1 / 5$ & $1 / 5$ & - & - & - & - & - & - & - & $1 / 5$ & - & $1 / 5$ & $1 / 5$ & P.I. \\
\hline $\mathrm{V} \quad 38 \mathrm{~B}$ & $1 / 5$ & $1 / 5$ & - & - & $1 / 5$ & - & - & - & $I / 5$ & - & - & - & - & P.I. \\
\hline$v 513$ & $1 / 5$ & $1 / 5$ & - & $1 / 5$ & $1 / 5$ & - & - & - & - & - & - & - & - & P.I. \\
\hline$v 581$ & - & $1 / 5$ & - & - & $1 / 5$ & $1 / 5$ & $1 / 160$ & $1 / 160$ & $\begin{array}{c}\text { L.P.B } \\
+\end{array}$ & & & & & \\
\hline V 517 & $1 / 5$ & $1 / 5$ & - & $1 / 5$ & - & $1 / 5$ & $1 / 20$ & - & $1 / 5$ & $1 / 5$ & - & - & - & P.I. \\
\hline V 582 & $1 / 5$ & $1 / 5$ & - & - & $1 / 5$ & $I / 5$ & $1 / 5$ & - & - & - & - & $1 / 20$ & $1 / 10$ & $\begin{array}{l}\text { séquestre } \\
\text { (noix) }\end{array}$ \\
\hline V 595 & $1 / 5$ & $1 / 5$ & - & - & - & - & - & - & $I / 5$ & $1 / 20$ & $1 / 20$ & $1 / 20$ & $1 / 5$ & P.I. \\
\hline
\end{tabular}

\footnotetext{
L.P.B. : mort avec lésions péripneumoniques

P.I. : poumon indemne
}

l'expérience, ne présente à l'autopsie qu'un séquestre de la grosseur d'une orange. Néanmoins $M$. mycoides est isolé des ganglions trachéo-bronchiques.

\section{c) Evolution des animaux vaccinés}

L'évolution sérologique figure dans le tableau 16 (déviation du complément Kolmer). Sur les 10 bovins vaccinés, un est mort de péripneumonie 63 jours après le début de la mise en contact après avoir présenté une évolution sérologique traduisant l'existence de lésions (V 581). Les 9 autres ont été sacrifiés après 96 jours de contact infectant: 7 révèlent un appareil pulmonaire indemne, un présente des adhérences pleurétiques au niveau de la paroi costale (V 509); leur origine semble fort ancienne et la péripneumonie n'est pas à coup sûr en cause, le dernier (V 582) offre un séquestre encapsulé de la grosseur d'une noix. Les ganglions trachéo-bronchiques de ces 9 bovins, ensemencés, ne donnent aucune culture de $M$. mycoides.

\section{CONCLUSION}

Sur les 10 bovins vaccinés depuis quatorze mois avec le vaccin lyophilisé $T_{1}$ et mis en contact avec des animaux infectants, un a succombé de péripneumonie bovine deux mois après le début de l'expérience, un présente un séquestre encapsulé minime et les huit autres peuvent être considérés comme indemnes de toute atteinte de la maladie.

\section{CONCLUSION GENERALE}

Les résultats qui viennent d'être énoncés permettent de garantir une immunité d'au moins un an à la suite de l'immunisation de bovins par le vaccin lyophilisé $T_{1}$, préparé dans les conditions retenues par le Laboratoire de Dakar.

En 1970, une expérience analogue à celle qui vient d'être décrite sera poursuivie pour apprécier la valeur de l'immunité conférée par un vaccin mixte péripneumonie bovine - peste bovine ( $\mathrm{T}_{1}$ streptomycino-résistant - souche de culture cellulaire RP OK BK 99).

Institut d'Elevage et de Médecine vétérinaire des Pays tropicaux, Maisons-Alfort.

Laboratoire national de l'Elevage et de Recherches vétérinaires, Dakar-Hann. 


\section{SUMMARY}

Quality of the immunity produced hy a CBPP freeze-dried vaccine prepared with the $T_{1}$ strain

During 1968 and 1969 , a series of experiments to test the immunity produced by a freeze-dried vaccine prepared with the $\mathbf{T}_{1}$ strain of $\mathrm{Myco}$ plasma mycordes (44th passage) has been carried out in the Laboratory of Dakar. The in-contact method described by the australjans workers has been utilized. Three and four months and half after vaccination the immunity is complete; fourteen months after immunization, 90 per cent of the animals are still protected In those conditions, a minimum protection period of one year is warranted.

\section{RESUMEN}

Valor de la inmunidad producida por una vacuna contra la perineumonia, liofilizada, preparada mediante la cepa $\mathbf{T}_{2}$

Durante los ar̃os 1968 y 1969, se efectuó, en el Laboratorio de Dakar, una serie de pruebas para determinar el valor de la inmunidad producida por una vacuna liofilizada preparada mediante la cepa $T_{1}$ (440 pasaje). Se utilizó el método llamado de contacto descrito por los buscadores de Australia. Es total la inmunidad tres meses y siete y media meses despues de la vacunación. Sigue de 90 p. 100 , catorce meses después de la inmunizacion. En estas condictones, se puede garantir un periodo mínimo de protección de un año.

\section{BIBLIOGRAPHIE}

1. BROWN (R. D.), "Endobronchial inoculation of cattle with various strains of Mycoplasma mycoides and the effects of stress », Res. ret. Sci, 1964, 5, 393-404.

2. BROWN (R.D.), GOURLAY (R. N.) et MacLEOD (A. K.), "The production of $\mathrm{T}_{1}$ broth culture contagious bovine pleuropneumonia vaccine ", Bull. epiz. Dis. Afr. 1965, 13, 149-155.

3. CAMPBELL (A. D.), « A preliminary note on the experimental reproduction of bovine pleuropneumonia ", J. Counc Sc1 Ind. Res., 1938, II, (2), 103-111.

4. DAVIES (G.), « Note on the $T_{1}$ broth vaccine for the inaugural technical conference of the JP:15/ phase IV Rinderpest Campangn ». Nairobi (Kenya) - November 1968.

5. DAVIES (G.), MASIGA (W. N.), SHIFRINE (M.) et READ (W.C.S.), " The efficacy of $T_{1}$ strain broth vaccine against CBPP: prelimunary incontact trials », Vet. Rec., 1968, 83, 239-244.

6. DAVIES (G.) et GILBERT (F. R.). "Vaccination contre la péripneumonie contagieuse bovine dans l'Est africain *, XXXVII ${ }^{\mathrm{e}}$ session générale du Comité de l'O 1.E, Paris, 1968.

7. DAVIES (G.), "The persistence of Mycoplasma mycoides in the host after vaccination with $\mathbf{T}_{1}$ broth vaccine ", Res. iet. Sci., 1969, 10, 225-231

8. DOUTRE (M. P.). «Valeur de l'immunité conférée par deux vaccins lyophilisés préparés à l'aide des souches KH 3 J et $T_{1}$ ", XXXVI ${ }^{e}$ session générale du Comité de l'O.I.E., Paris, 1969.

9. HUDDART (J. E.), «Outline of work on CBPP in Tanzaria, 1964 to 1966 ", Working paper $n^{\circ} 18$, $3^{\text {e }}$ Réunion des Experts de la Péripneumonie bovine F.A.O./O.I.E./O.A.U., Khartoum, 1967.

10. HUDSON (J R.) et TURNER (A.W.), « Contagious bovıne pleuropneumonia. - A comparison of the efficiency of two types of vaccine ", Aust. vet. J., 1963, 39, 373-385.

11. HUDSON (J.R.), «Contagious bovine pleuro pneumonia. - The immunizing value of the attenuated strain KH $3 \mathrm{~J}$ \%, Alust. vet. f., 1965, 41, 4349.

12. HUDSON (J. R.), «Contagious bovine pleuropneumonia. - Experiments on the susceptıbility and protection by vaccination of different types of cattle », Aust. ret. J., 1968, 44, 83-89.

13. PROVOST (A.), « Rapport sur les recherches entreprises sur la péripneumonie bovine au Laboratoire de Recherches vétérinaires de Farcha, FortLamy (Tchad), de 1964 à $1966 \%$, 3* Réunion du Groupe d'Experts F.A O./O.I.E./O.A.U. sur 1a péripneumonie bovine. 12-15 février 1967.

14 a Rapport de la 3 * Réunion des Experts de la Péripneumone bovine F.A.O./O.I.E./O.A.U, », Khartoum, 1967.

15. REGNOULT (M.) et ORUE (J.), «Valeur de la méthode d'snfection expérimentale à Mycoplasma mycoides par intubation intra-bronchique pour l'obtention de malades et de porteurs de germes», $2^{2}$ Réunion des Experts de la Péripneumonie bovine F.A.O./O.I.E./C.C.T.A., Muguga, 1964.

16. SHIFRINE (M), STONE (S.S.) et DAVIES (G.) «CBPP : serologic response of cattle after single and double vaccination with $T_{1}$ culture vaccine $»$, Rev. Elev. Méd. vét. Pavs trop., 1968, 21, (1), 49. 58. 